\title{
High Grade Salivary Gland Carcinoma
}

National Cancer Institute

\section{Source}

National Cancer Institute. High Grade Salivary Gland Carcinoma. NCI Thesaurus. Code C8018.

A usually aggressive salivary gland carcinoma with high grade histopathologic features. It includes the salivary duct carcinoma, salivary gland oncocytic carcinoma, and high grade salivary gland mucoepidermoid carcinoma. 